\title{
Nanoselenium Enhanced Wheat Resistance to Aphids by Regulating Biosynthesis of DIMBOA and Volatile Components
}

chunran zhou

China Agricultural University

dong Li

China Agricultural University

xinlei shi

China Agricultural University

jingbang zhang

China Agricultural University

quanshun an

China Agricultural University

yangliu wu

China Agricultural University

lu kang

China Agricultural University

jia-qi Li

China Agricultural University

Canping Pan ( $\square$ canpingp@cau.edu.cn )

China Agricultural University

\section{Research}

Keywords: wheat, aphids, plant resistance, nanoselenium, metabolite pathway

Posted Date: August 25th, 2021

DOl: https://doi.org/10.21203/rs.3.rs-824089/v1

License: (c) (i) This work is licensed under a Creative Commons Attribution 4.0 International License. Read Full License

Version of Record: A version of this preprint was published at Journal of Agricultural and Food Chemistry on November 17th, 2021. See the published version at https://doi.org/10.1021/acs.jafc.1c05617. 

1 Nanoselenium enhanced wheat resistance to aphids by regulating

2 biosynthesis of DIMBOA and volatile components

3 Chunran Zhou ${ }^{\text {a, }}$, Dong Li ${ }^{\text {a, }}$, Xinlei Shi ${ }^{\text {a }}$, Jingbang Zhang ${ }^{\text {a }}$, Quanshun An ${ }^{\text {a }}$, Yangliu Wu ${ }^{\text {a }}$, Lu

$4 \quad$ Kang ${ }^{\mathrm{a}}$, Jia-Qi $\mathrm{Li}^{\mathrm{a}}$ and Canping Pan ${ }^{\mathrm{a}, *}$

5 Innovation Center of Pesticide Research, Department of Applied Chemistry, College of

6 Science, China Agricultural University, Beijing 100193, China Yuanmingyuan West Road 2,

7 Beijing 100193, PR China

8

$9 \quad{ }^{1}$ Chunran Zhou and Dong Li contributed equally to this paper.

$10 *$ Corresponding author

11 Canping Pan: canpingp@cau.edu.cn 


\section{Abstract}

Aphids are one of the most destructive insects in many cultivated plants including wheat, which can cause significant yield loss and damage the quality of agricultural products. Therefore, it is essential to control the occurrence of aphids during wheat growth. Previous studies reported the alterations in the resistance of wheat to pests induced by several external factors such as nutrients in soils and nano-carbonaceous materials. In this study, nanoselenium (nano-Se) was sprayed on wheat leaves at several concentration levels (1.0, 5.0, and $20 \mathrm{mg} / \mathrm{L})$. Nano-Se $(5.0 \mathrm{mg} / \mathrm{L})$ could significantly reduce Sitobion avenae number (36\%) compared with the control. The foliar application of nano-Se was found to enhance the antioxidation capacity by reducing MDA concentration and increasing GSH-Px, CAT, GSH, Pro and VE concentrations. Phenylpropane pathway was activated after the application of nanoSe, with significantly increasing apigenin and caffeic acid concentrations. The highlevel expression of the related genes (TaBx1A, TaBx3A, TaBx4A, TaASMT2, and TaCOMT) induced the increasing melatonin concentration by $88.6 \%$ and DIMBOA concentration by $64.3 \%$. Different ratios of the secondary metabolites to nano-Se were conducted to examine the effects on wheat resistance to the Sitobion avenae. The results revealed the combination of nano-Se and melatonin can achieve the best overall performance by reducing the Sitobion avenae infection by $52.2 \%$. The results from this study suggest that the coordinative applications nano-Se and melatonin combination could be more effectively improve the wheat resistance to aphids via promotion of volatile organic compound synthesis and modulation in phenylpropane and indole metabolism pathways.

Keywords: wheat, aphids, plant resistance, nanoselenium, metabolite pathway. 


\section{Introduction}

Wheat (Triticum aestivum L.) is one of the most widely grown grain crops in the world. It could be vulnerably attacked by various herbivores during the process of growth. Aphids are common agricultural spines suck pests, which have a relatively short reproduction cycle hence large population. They directly ingest the nutrients present in plant phloem screen, and can act as the transmission vector of plant viral diseases [1]. These activities can seriously affect the crop yields and the quality of grains [2]. Pesticides are the most widely used to control aphids; however, the overuse of chemical pesticides could lead to potential environmental contamination and damage ecosystem services [3]. Integrated pest management has been strategically adopted to control aphids with biological controls including insect-resistant varieties, crop rotation [4], natural enemy [5], and RNA interference, in many cases they fail to achieve satisfactory performance due primarily to short persistence and high costs [6]. Therefore, it is urgent to develop green, safe, and ecologically reasonable methods for the prevention and control of aphids.

Intervention factors such as plant hormones and nanomaterials play a vital role in plant defense against insect pests [7]. Qi et al. showed jasmonic acid (JA) and ethylene contents were increased by Melon aphid infestation, which enhanced the resistance of cucumber plants to aphids [8]. Pawel et al. demonstrated that luteolin, quercetin and apigenin in winter triticale can reduce hydrogen peroxide and superoxide radical concentration, protecting the winter triticale from the infestation of Sitobion avenae F and beetles [9]. Many allelopathic substances such as 2,4dihydroxy-7-methoxy-1,4-benzoxazin-3-one (DIMBOA) is commonly found in wheat, corn, and other gramineous crops in response to environmental changes [10]. DIMBOA and its degradation products manifested strong inhibitory effects on European corn borer in wild oat and ryegrass [11]. DIMBOA can diminish the reproduction rate of aphids[12]. Varsani et al. reported that DIMBOA, derived from benzoxazinoid $(\mathrm{BX})$ in maize, is involved in callus accumulation with the effects to 
enhance the plant resistance to aphids [10]. Melatonin (MT) can regulate the levels of reactive oxygen species (ROS) in plants, thereby maintaining cell homeostasis and promoting plant growth [13]. Recently, Bapat et al. found that silica nanoparticles $(0.2 \mathrm{mg} / \mathrm{mL})$ application significantly reduced insect weight and inhibited the Helicoverpa armigera growth in tomato plants [14].

Selenium is the main component in the antioxidant enzyme glutathione peroxidase (GSH-Px) and plays an important role in oxidative defense [15]. Zhang et al. found that sodium selenite could regulate the indole metabolism by alleviating imidacloprid-induced stress in garlic [16]. Compared with inorganic and organic Se, nano-Se demonstrated high bioavailability, low toxicity, and strong free radical scavenging ability [17]. Foliar applied nano-Se can enhance flavonoids and phenolic acids concentration in strawberries and alleviate the associated salt stress [18]. NanoSe was found to be able to enhance the efficiency of antioxidants and improve the metabolism of amino acids to inhibit the growth of $S$. sclerotiorum in oilseed rape [19]. Mechora et al. showed that the highly accumulated Se could protect Stanlega pinnata and Astragalus bisulcatus against flower thrips and two-spotted spider mite [20]. Interestingly, it was observed that multiple Se species could increase melatonin concentration in plants; Se and melatonin combination could alleviate biological and abiotic stress by regulating plant primary and secondary metabolism [21], [22]. However, little is known about the mechanism of the increased plant resistance to insects induced by Se treatment and the related indoles alkaloids and phenylpropane pathways.

Volatile organic compounds (VOCs) are of great potential in plants to defense against insects. Plants respond to herbivores through multiple defensive modes, and the formation of VOCs is commonly an immediate and important response [23]. Mitra et al. found the formation of high levels of benzyl alcohol, 1,3-diethylbenzene, acetophenone, 1-nonanol, ethyl acetophenone, and1-hexanol was associated with the decrease in aphids numbers [24]. Accumulation of S-linalool and (E)- $\beta$-caryophyllene could alter insect communities by attracting the natural enemies thus repelling the 
brown planthoppers from rice plants [25]. Delaney et al showed that cis-jasmone enhanced wheat resistance to leaf beetles by attracting natural enemies [26]. The applied silicon could attract aphid natural enemies in wheat by enhancing the formation of volatile compounds (geranyl acetone) [27]. The accumulated (E)- $\beta$ farnesene and methyl salicylate could decrease the aphid abundance, which could correspondingly improve the wheat resistance to aphids [28]. Element selenium can inhibit the growth and reproduction of the myzus persicae, weevils, cabbage root flies, beetles, caterpillars, and crickets by accumulating the VOCs contents [29]. Our twoyear field trial study showed that the foliar application of nano-Se can effectively alleviate the pesticide-induced oxidative stress by improving the levels of secondary metabolites and aroma compounds in tea plants [30]. However, the role of nano-Se in the mechanism of increasing VOCs and wheat resistance to aphids remains largely unclear.

This study aimed to investigate the potential mechanism of the enhanced resistance of wheat to aphids after foliar application of nano-Se. Changes in antioxidant ability and the concentration of flavonoids, phenolic compounds, phytohormone and VOCs were measured in the wheat seedlings. The optimal proportions and composition of these metabolites, as well as the amount of nano-Se application, were examined based on the defense of wheat plants to Sitobion avenae. It is concluded that the combination of nano-Se and melatonin could be involved in the resistance mechanism mainly by regulating the VOCs, phenylpropane, and indoles alkaloid pathways.

\section{Materials and methods}

\section{Plant growth}

The wheat plants used in the experiments were grown in the intelligent greenhouse of the College of Agriculture, China Agricultural University (Beijing, China). Wheat seeds (Liaochun10) were soaked in $0.5 \%$ sodium hypochlorite for 30 min and then cleaned five times with distilled water. The wheat seeds were planted in 
plastic pots (14 cm in diameter and $11 \mathrm{~cm}$ in height) containing organic soil (nutritional soil: peat: vermiculite $=4: 1: 1$ ). During the whole experiment period, the plants maintained the relative humidity at $70 \%$ at the day/night temperature of $25 / 20{ }^{\circ} \mathrm{C}$ with a day/night cycle of $14 / 10 \mathrm{~h}$ and the light intensity at $80 \mu \mathrm{mol} \mathrm{m} \mathrm{m}^{-2} \mathrm{~s}^{-1}$. The nano-Se $(50-78 \mathrm{~nm})$ applied in this study was characterized and reported in our previous study [31]. The plant experiment was separated into four groups including three levels of nano-Se treatment $(5,10$, and $20 \mathrm{mg} / \mathrm{L})$ and the controls. These four treatments each had five duplicates.

\section{Aphid Infestation}

Aphid (Sitobion avenae) was provided by Dunlun Song, College of Plant Protection, China Agricultural University. The aphids were kept in the compartment with the relative humidity at $50 \% \sim 70 \%$, temperature at $18 \sim 25^{\circ} \mathrm{C}$, and the light intensity of $80 \mu \mathrm{mol} \mathrm{m}^{-2} \mathrm{~s}^{-1}$. The nano-Se was applied in the wheat seeding stage. Ten adults of apterous S. avenae were then inoculated on wheat leaves after two days. Nano-Se was sprayed every 7 days and used 3 times at all. Aphid numbers were counted every 5 days after the inoculation (only adults were counted). All experiments were carried out on 120 mesh nylon mesh $(75 \times 75 \times 75 \mathrm{~cm})$, and there were five nylon meshes in total. Each nylon mesh had four different treatments (control, Se1, Se5, and Se20) with five replicates. The whole test lasted for one month. The aphid was removed from the wheat leaves and collected the wheat seedlings in liquid nitrogen. The samples were stored at $-80^{\circ} \mathrm{C}$ before measurement for antioxidant enzymes and gene expression.

\section{Determination of antioxidant capacity}

Superoxide dismutase (SOD), catalase (CAT), ester oxygenase (LOX), glutathione peroxidase (GSH-Px), polyphenol oxidase (PPO), peroxidase (POD), ascorbate peroxidase (APX), glutathione (GSH), proline (Pro), malondialdehyde (MDA), vitamin e (VE), and lipid peroxidation (LPO), which were measured using the commercial kits from Nanjing Jiancheng Bioengineering Institute (Nanjing, China). 
The concentrations of JA and salicylic acid (SA) in wheat seedlings were measured using the methods developed in our laboratory [30]. Briefly, wheat seedlings powder $(100 \mathrm{mg})$ was extracted with $1 \mathrm{~mL}$ of solution $(80 \%: 19 \%: 1 \% \mathrm{~V} / \mathrm{V} / \mathrm{V}$, methanol: water: formic acid). The solutions were used ultrasound for $10 \mathrm{~min}$ and then centrifuged at $12000 \mathrm{rpm}$ for $10 \mathrm{~min}$. All supernatants were blown dry with nitrogen and made a volume of $100 \mu \mathrm{L}$ with $80 \%$ methanol in water. The specific parameters of JA and SA were supplied in Table S1.

DIMBOA was measured using the method reported by Kong et al [32]. The frozen wheat leaves were ground, and the powder $(100 \mathrm{mg})$ was extracted with 1.0 $\mathrm{mL}$ methanol containing $1 \%$ formic acid. The mixture solutions were using ultrasound in a cold bath for $5 \mathrm{~min}$, then overnight extracted in a refrigerator at $4^{\circ} \mathrm{C}$. The next day, the treated samples used ultrasound for $5 \mathrm{~min}$ at $4^{\circ} \mathrm{C}$, then centrifuged at $10,000 \mathrm{rpm}$ for $5 \mathrm{~min}$. The above method was repeated three times and all supernatants were collected. The supernatants were blown dry with nitrogen gas and made up the final volume at $1 \mathrm{~mL}$ (methanol: water: acetic acid=59: 40: 1, V/V/V). The solutions were filtered with a $0.22 \mu \mathrm{m}$ nylon syringe. Acetonitrile and formic acid water (containing $0.1 \%$ formic acid, $\mathrm{V}: \mathrm{V}=38: 62$ ) were used as mobile phases $\mathrm{A}$ and $\mathrm{B}$ for isocratic elution on a reversed-phase C18 column $(4.6 \mathrm{~mm} \times 150 \mathrm{~mm}, 5 \mu \mathrm{m})$, respectively. The flow rate was $1.0 \mathrm{~mL} / \mathrm{min}$ at a wavelength of $280 \mathrm{~nm}$ on an HPLC1260 instrument equipped with a UV detector.

The determination of melatonin content was modified based on Li et al [21]. In short, wheat leaves $(100 \mathrm{mg})$ were ground and then added $1 \mathrm{~mL}$ solution (acetonitrile: hexane $=80: 20, \mathrm{~V}: \mathrm{V})$. The mixture was violently shacked for $5 \mathrm{~min}$. It was used ultrasound for $30 \mathrm{~min}$ at $30^{\circ} \mathrm{C}$ and centrifuged for $5 \mathrm{~min}$ at $12000 \mathrm{rpm}$. They were collected by repeating the twice operating method. All supernatants were blown dry with nitrogen and the residues were extracted by $1 \mathrm{~mL}$ solution. These solutions were purified in a $2 \mathrm{~mL}$ centrifuge tube containing $20 \mathrm{mg}$ MWCNT. After that, it was shacked for $2 \mathrm{~min}$ and centrifuged for $1 \mathrm{~min}$ at $10000 \mathrm{rpm}$. The concentration was filtered with a $0.22 \mu \mathrm{m}$ nylon syringe. The experiment was analyzed by high-performance liquid 
chromatography-tandem mass spectrometry (HPLC-MS/MS) equipped with an electrospray ionization (ESI) source operating in positive mode (Agilent 6410B triple quadrupole). Acetonitrile and formic acid water (contain $1 \%$ formic acid) were used as mobile phases A and B for isocratic elution, respectively. The injection volume was $5 \mu \mathrm{L}$ and the flow rate was $0.2 \mathrm{~mL} / \mathrm{min}$. Other optimized information was applied in Table S1.

\section{Analysis of flavonoids and phenolic acid compounds}

Briefly [31], wheat leaves (100mg) were ground to the powder in liquid nitrogen. They were added $1 \mathrm{~mL}$ water (containing $60 \%$ ethanol) in a centrifuge tube. The solution was used ultrasound for $30 \mathrm{~min}$ at $30^{\circ} \mathrm{C}$ and then centrifuged for $5 \mathrm{~min}$ at $12000 \mathrm{rpm}$. The concentration was collected by conducting twice operating method. The mixture was collected and blown dry with nitrogen. The final volume was $1 \mathrm{~mL}$ with the above solution and centrifuged for $1 \mathrm{~min}$ at $10000 \mathrm{rpm}$. It was purified with $100 \mathrm{mg} \mathrm{C} 18$ in $2 \mathrm{~mL}$ centrifuge tube, shake for $2 \mathrm{~min}$ and centrifuged for 1 minute at $10000 \mathrm{rpm}$. Next, the supernatant was filtered into the injection vial by $0.22 \mu \mathrm{m}$ nylon syringe. HPLC-MS/MS (Agilent 6410B triple quadrupole) was used to analyze the concentration. The parameter was showed in Table S2 and Table S3.

\section{Verification with significant difference of exogenous}

Based on the above results (plant hormone, flavonoids and phenolic acids), JA, SA, MT and API were selected to test their effect on wheat seedlings growth. Different concentration $(0,1,5,10$ and $50 \mathrm{mg} / \mathrm{L})$ of each compound was sprayed to the wheat seedlings. Among the 17 treatments, wheat plant height, root length, fresh weight, and dry weight, along with phytohormone and antioxidant capacity, were measured and showed Figure S1-S4. MT at the applied rate of $10 \mathrm{mg} / \mathrm{L}$ demonstrated showed the highest impacts on the wheat seedling growth based on the observed results (Figure S1S4). Next, nano-Se (5mg/L) and MT (10mg/L) were tested for their effects on S. avenae. Aphids numbers and changes of wheat compounds were measured. Each treatment was set up with five replicates. Wheat growth conditions and aphid infestation were same as the before. 


\section{RNA Extraction and Real-Time Quantification}

Four groups of wheat plants were prepared for RNA-seq. The total RNA was extracted using RNA pure Plant Kit (Tiangen Biotech, Beijing, China). Then, total RNA was reverse transcribed to cDNA according to instructions of the manufacturer (TURE script 1st Stand cDNA SYNTHESIS Kit). The RT-qPCR was performed using a CFX 96 PCR system (Bio-Rad) with Super Real PreMix Plus Kit (SYBR Green).

\section{Detection of volatile components in wheat seedlings}

Compared with other groups, the combination of nano-Se $(5 \mathrm{mg} / \mathrm{L})$ and MT $(10 \mathrm{mg} / \mathrm{L})$ showed a better effect on reducing of aphid number. Gas ChromatographyIon Mobility Spectrometer (GC-IMS) was used to analyze VOCs released from the wheat seedlings. About $0.5 \mathrm{~g}$ wheat seedlings samples were weighted in a $20-\mathrm{mL}$ headspace vial. The vials were equilibrated at $60^{\circ} \mathrm{C}$ for $20 \mathrm{~min}$ before headspace injection. Table S4 (the analysis conditions of GC-IMS unit) and Table S5 (gas chromatographic conditions) have specific parameters.

The analysis software includes VOCAL and three plugins, which can analyze a sample from a different angle.1) VOCAL was used to qualitative and quantitative the data and spectrogram. The software equipped with NIST and IMS database was used for qualitative analysis of the substance. Each point in the figure stands for one volatile organic compound, which can be quantitatively analyzed by establishing a standard curve. 2)Reporter plugin was used to compare the spectrogram difference between the samples directly (three-dimensional spectrogram, two-dimensional top view, and differential spectrogram).3) Gallery Plot plugin: fingerprint comparison, quantitative and visual compare the difference of volatile organic compounds between different samples.4) Dynamic PCA plugin: dynamic principal component analysis, which was used for cluster analysis of samples and rapidly determined the unknown samples.5)

“Nearest neighbor" fingerprint analysis: this feature can rapid comparison of samples based on the strength of compounds in the selected region.

\section{Statistical analysis}

GraphPad Prism version 7.0 was used to draw graphs. One-way analysis of 
variance was performed using SPSS 26.0. Differences among mean values $(p<0.05)$ of each treatment were compared with t-test.

\section{Results}

\section{Effects of nano-Se on S. avenae growth}

The inhibitory effects of nano-Se on $S$. avenae growth are presented in Table 1 . Nano-Se could significantly reduce the number of $S$. avenae. Compared to the controls, nano-Se $(5.0,10.0,20.0 \mathrm{mg} / \mathrm{L})$ reduced the aphid numbers by $36.4 \%, 26.2 \%$, $11.5 \%$, respectively (Figure 1). Nano-Se $(5 \mathrm{mg} / \mathrm{L})$ demonstrated the best showed better inhibition effects among the three all treatments.

\section{Effects of different concentrations of nano-Se on phytohormone in wheat} seedlings

As shown in Figure 2A-D, nano-Se application (5.0, 10.0, and $20.0 \mathrm{mg} / \mathrm{L}$ ) could promote the increase in JA, MT, and DIMBOA content in many cases, but reduce the SA content in wheat. Compared with the control, nano-Se at the application rate of 5.0 $\mathrm{mg} / \mathrm{L}$ could enhance the JA, MT and DIMBOA concentration by $150 \%, 88.6 \%$, and $64.3 \%$; at the same time, SA concentration decreased by $88.0 \%$.

\section{Antioxidant capacity of wheat seedlings with nano-Se treated}

The application of nano-Se can alter the antioxidant capacity of wheat seedlings as measured by antioxidant enzymes and some related chemicals (Figure 3). Among the three levels of nano-Se treatments, the concentration at $5.0 \mathrm{mg} / \mathrm{L}$ manifested the greatest impacts. Antioxidant enzyme activity APX, LOX, GSH-Px, CAT, and POD increased by 4.1 times, 3.1times, $69.2 \%, 38.5 \%$, and $10.7 \%$, respectively. The concentration of GSH, Pro, and vitamin E (VE) contents enhanced by 45.9\%, 19.0\%, and $44.2 \%$, respectively (nano-Se $5 \mathrm{mg} / \mathrm{L}$ ). As for MDA (Figure $3 \mathrm{H}$ ), the concentration decreased by $13.6 \%$. The trend is that the application of nano-Se at 5.0 $\mathrm{mg} / \mathrm{L}$ could significantly improve the antioxidant capacity of wheat seedlings by increasing the activity of antioxidant enzymes and the related chemical concentration. Effects of nano-Se application on flavone compounds and phenolic acids in wheat 


\section{seedlings}

Flavone compounds and phenolic acids play an important role in plants to defense against pests and disease [9]. In general, the concentration of flavone compounds and phenolic acids increased with nano-Se treatment, especially at the application rate of $5.0 \mathrm{mg} / \mathrm{L}$ (Figure 4). The flavone compounds measured in this study included quercetin, luteolin, apigenin, rutin, kaempferol and myricetin which all increased to varying degrees after application of nano-Se. Among these compounds, luteolin demonstrated the greatest increase, increased by 1.7 times, 1.3 times, and 1.1 time after the application of nano-Se at 1.0, 5.0, and $20.0 \mathrm{mg} / \mathrm{L})$. Nano-Se $(5 \mathrm{mg} / \mathrm{L})$ increased apigenin, quercetin, and rutin levels by 1.0 times, $14.5 \%$, and $22.2 \%$, respectively. However, the nano-Se application had no obvious effects on kaempferol and myricetin.

As for the phenolic acids, compared to the controls, the application of nano-Se at $5.0 \mathrm{mg} / \mathrm{L}$ could lead to a significant increase in the concentration of caffeic acid by 74.9\%, hydroxy-3, 5-dimethoxycinnamic acid by 73.6\%, 4-hydroxybenzoic acid by 98.0\%, 4-hydroxycinnamic acid by $38.0 \%$, and chlorogenic acid by $14.7 \%$. However, the nano-Se application had no obvious effects on ferulic acid, syringic acid, and vanillin acid contents. The results indicate that the application of nano-Se at $5.0 \mathrm{mg} / \mathrm{L}$ could invoke the changes in the concentration of plant secondary substances in wheat seedlings.

\section{Effects of significant difference of exogenous on $S$. avenae growth}

As shown in Figure 5 A, compared to control, aphid number reduced by $42.9 \%$ (nano-Se5), 35\% (MT10), and 52.2\% (the combination of nano-Se5 and MT10), respectively. The nano-Se application resulted in an increase in DIMBOA, MT, and JA concentration by $31.1 \%, 57.0 \%$, and $43.6 \%$ respectively. The MT application caused increased DIMBOA, MT, and JA levels by $31.8 \%, 62.8 \%$, and $38.2 \%$, respectively. The combined application of nano-Se5 and MT10 enhanced the DIMBOA, MT, and JA concentration by $34.8 \%, 70.8 \%$, and $51.3 \%$, respectively. The SA contents decreased by $42.2 \%, 37.3 \%$, and $57.5 \%$ when treated with nano-Se, MT, 
and their combination (Figure 5B).

The expressions of DIMBOA (TaBx1A, TaBx3A, TaBx4A) and MT (TaASMT2, TaCOMT) related genes were determined (Figure 5C). Compared to the control, $\operatorname{TaBx} 1 A$ (2.5 times), TaBx3A (40.0\%), TaBx4A (55.9\%), TaASMT2 (34.1\%), and TaCOMT (12.3 times) were increased by nano-Se5. MT could cause the expression of TaBxl $A$ by 2.0 times, $T a B x 3 A$ by $2.0 \%, T a B x 4 A$ by $23.7 \%$, TaASMT2 by $8.4 \%$, and TaCOMT by 7.5 times. Their combination treatment increased the expression of TaBx1A, TaBx3A, TaBx4A, TaASMT2 and TaCOMT by 4.1 times, 52.5\%, 1.0 times, $52.3 \%$ and 25.3 times, respectively.

\section{Combination of nano-Se and melatonin affect the VOCs of wheat seedlings under aphid infection}

As can be seen in Figure 6A, four kinds of samples can separate. There was a significant difference among the four treatments based on the results of fingerprint similarity analysis, and collaboration of Se and MT was the greatest.

For convenient comparison, the top view is shown in Figure 6B. 1) The background of the spectrogram is blue, and the red vertical line at abscissa 1.0 is the RIP peak (reaction ion peak and normalized treatment).2) The vertical axis represents retention time(s) of the gas chromatograph, the abscissa represents the ion migration time.3) Each point on either side of the RIP peak represents a kind of volatile organic compound. White color means a low concentration, and red color means a high concentration. There was a difference in the types and concentrations of volatile organic compounds among all samples (Figure 6C). Therefore, the levels of volatile compounds were estimated according to the degree of the colors.

Overall, there were 45 VOCs detected in the wheat seedlings by GC-IMS. The identified VOCs included 6 alcohols, 10 ketones, 6 esters, 5 alkenes, 5 aldehydes, and 3 furans (Figure 7A). The distributions of the molecular fingerprints in the differently treated wheat samples (nano-Se, MT, the combination of nano-Se and MT, as well as the controls) were plotted in Figure 7B. The row in Figure 7B represents all the signal peaks from a specific sample, and the column represents the signal intensity of a 
specific VOC in different wheat samples. In addition, the numbers in the Figure represent the uncertainty of the identified substances in the migration spectrum library. The specific VOCs related to aphid resistance were plotted against the treatment of nano-Se, MT, and their combination as well as the control (Figure 7C).

\section{Discussion}

The infestation of aphids in plants can cause huge damage to crop production. Currently, exogenous interventions such as chemical pesticides are the most effective approach to control insect pests and achieve high crop yields [3]. However, the longterm use of pesticides could lead to insects developing resistance and cause the ineffectiveness of these chemicals. Activation of the plant itself anti-aphid system is considered a green protocol in plant protection and sustainable agricultural production. In this study, we demonstrated that the application of nano-Se could effectively reduce aphid populations in wheat seedlings (Table 1). This could be due to the mechanism that nano-Se could promote the synthesis of flavones, phenolic acids, plant hormones and the expression of the related genes, which could further influence the phenylpropane pathway and the metabolism of indole alkaloids. The application of nano-Se also increased antioxidants and VOCs concentrations, which promotes the resistance of wheat seedlings to aphids (Figure 8).

Plant secondary metabolites play an important role in the resistance to insect feedings. These chemicals commonly include phenolics (phenolic acids and flavonoids) and terpenoids (diterpenes and monoterpenes), which could attract specific insects and their natural enemies [33]. A previous study showed that Se hyperaccumulators could promote plant growth by protecting Artemisia ludoviciana and Symphyotrichum ericoides against grasshoppers [34]. The effect of Se on secondary metabolites was mainly based on abiotic stress, including salt, heavy metal, and drought [35]. However, few studies were conducted to elucidate the effects of Se on the formation of secondary metabolites (phenylpropane and indole alkaloid metabolism) and their impact on insects. Our previous study reported that foliar 
application of nano-Se $(10 \mathrm{mg} / \mathrm{L})$ could alleviate pesticide stress via regulating the ascorbate-glutathione cycle in tea trees [30]. In pepper plants, nano-Se $(20 \mathrm{mg} / \mathrm{L})$ could enhance phenylpropane and fatty acid metabolism [36], causing a significant increase in the concentration of flavonoids (by 80\%) and phenolic acids (by 65\%). In this study, the increased concentration of myricetin, rutin, apigenin, kaempferol, quercetin, caffeic acid, hydroxy-3, 5-dimethoxycinnamic acid, 4-hydroxybenzoic acid, 4-hydroxycinnamic acid, and chlorogenic acid was observed after the application of nano-Se $(5 \mathrm{mg} / \mathrm{L})$ to wheat seedlings with aphid infection (Figure 4). These metabolites of apigenin and caffeic acid were then selected to evaluate the growth and metabolism of wheat under aphid stress. This practice could enhance wheat growth including plant height, length, and weight, the concentrations of flavonoids (luteolin and quercetin), and phenolic acids (caffeic acid, 4hydroxybenzoic acid, and 4-hydroxycinnamic acid) in the seedlings (Figures S1, S2, and S3). The application of plant strengthener 4-fluorophenoxyacetic acid was found to suppress pest populations [37]. The enhanced resistance to insects could be due to the deposition of flavonoid polymers in plant cells. Czerniewicz's et al. found that the formation of luteolin, apigenin, caffeic acid, and 4-hydroxybenzoic acid compounds could impair insect feeding, thus protecting wheat from aphid infection [9].

In general, plants can establish a dynamic equilibrium of ROS via formation or removal processes depending on the growth conditions. Plant secondary metabolism and antioxidant system can synergistically improve plant resistance to biotic and abiotic stresses. In the present study, the antioxidant enzymes (CAT, POD, SOD, LOX, GSH-Px, and APX) and non-enzymes (VE, MDA, GSH, Pro, and LPO) system were found to change to varying degrees with nano-Se treatment (Figure.3). A previous study reported that sodium selenate $(5 \mu \mathrm{M})$ application could alleviate the salt stress of wheat seedlings by up-regulating the antioxidants and osmolytes metabolism [38]. Selenite present in soil $(0.5 \mathrm{mg} / \mathrm{kg})$ enhanced the antioxidant enzymes (CAT, POD, and PPO) and reduced the MDA concentration, forming a favorable situation in oilseed against Sclerotinia sclerotiorum stress [19]. Li et al. used 
$200 \mu \mathrm{M}$ of Se treatment to inhibit silkworms via significantly decreasing their growth and reproduction [39]. In addition, sodium selenate $(6.5 \mu \mathrm{M})$ after foliar application can effectively protect rice against $N$. lugens infestation [40]. Sodium selenate has been shown to effectively inhibit the growth of insect pests in crops, but without elucidating the underneath mechanism [41],[42]. Our study suggests that nano-Se application could alleviate the effects of herbivores by regulating the secondary metabolism and antioxidative ability.

Phytohormone was considered as the first line to defense against insect pests the insects [43]. The JA, SA, and MT signaling was intensively involved in herbivore defense in plants. In the present study, the application of nano-Se $(5,10$, and $20 \mathrm{mg} / \mathrm{L})$ could lead to the increase in JA, MT, and DIMBOA concentration, while the SA concentration was reduced (Figure.2). The remarkable effects were observed on MT and SA concentration changes, these two phytohormones were selected to apply directly on wheat seedlings to evaluate the plant metabolism. The growth parameters (height, length, and weight) and phytohormones (JA, SA, DIMOBA and MT) were measured in wheat seedlings (Figure. S1 and S4). JA and other signaling pathways interact to regular and enhance plant resistance to insects [44]. It has been demonstrated that the ROS and hormonal signaling were integrated to respond to insect herbivores [45]. Silicon could also regulate the biosynthesis of phytohormones (JA, SA and IAA) and reduce the concentration of ROS; these two processes together enhance plant resistance to herbivorous insects [46]. In our study, nano-Se could activate the indole alkaloid pathway and the related genes, which correspondingly increased the MT and DIMBOA contents. Extensive research demonstrated that MT is involved in regulating circadian rhythms in aphids [47]. The signal alternatively activates the molecular pathway that leads to the reproduction and migration responses. MT application $(50 \mu \mathrm{M})$ could induce the antioxidant enzymes activities (PPO, CHI, and PAL), thus regulating the ROS concentration in plants [48]. Furthermore, MT treatment increased MeJA concentration and up-regulated the JA biosynthesis genes, as a result, it enhanced the tomato defense against disease. MT is 
found to regulate flavonoids and carotenoid biosynthesis as well [49], promote the synthesis of amino acids, organic acids and sugars, and enhance the plant resilience under biotic (fungi, bacteria, and insects) and abiotic (heat, cold, drought, and salt) stresses [50]. DIMBOA is an allelochemical and can react with glucose to enhance resistance to aphids by promoting the callose deposition in maize [51]. DIMBOA (0.1 $\mathrm{mM}$ ) could reduce the Sitobion avenae F numbers to levels below the economic damage threshold [12]. However, the anti-aphid mechanism related to nano-Se and DIMBOA was not elucidated. In this study, the combination of nano-Se and MT application increased DIMBOA and MT, as well as the levels of the related genes which could be associated with the indole alkaloid pathway, thereby could decrease the aphid numbers in the wheat seedlings.

In this study, the release number of VOCs increased in the wheat seedlings treated by nano-Se. GC-IMS analysis revealed the increased concentration of ethanol, 1-popanol, Isopentyl alcohol, 2-butanone, 2-heptanone, acetoin, acetone, benzaldehyde, ethyl 2-methylpropanoate, and ethyl acetate dimer in the presence of nano-Se bio-fortification. However, 2-pentenal (E) dimer, hexanal monomer, ethyl hexanoate, and phenylacetaldehyde concentrations were reduced. Plants can produce a wide array of volatile metabolites, including terpenes, fatty acid derivatives, aromatic compounds, and amino acid derivatives [52]. The increased concentration of benzaldehyde, acetoin, and 1-propanol facilitated the repelling response to herbivorous insects in sorghum seedlings [53]. Zhuang et al. found that the volatile indoles increased with insect feeding; these chemicals could improve the resistance in rice plants [54]. Zhang et al. showed that VOCs including acetone, hexanal, and pentalamide play a vital role in impacting insect behaviors [55]. For example, VOCs could function as either attractants or antifeedants, and effectively control insect activities. It is commonly accepted that VOCs and insect pheromones could be combined to regulate insect behaviors. Our present study found that the combinations of nano-Se with phytohormones (MT and DIMBOA) can markedly reduce the insect numbers most likely due to the increasing VOCs in wheat seedlings. 


\section{Conclusion}

In this study, the foliar application of nano-Se could effectively decrease the aphid number compared with the control. The corresponding insect-resistant mechanism could be associated with the increasing levels of secondary metabolites (API and caffeic acid) and plant hormones (MT and DIMBOA) in the indole alkaloid and phenylpropane metabolisms in wheat seedlings. Different ratios of nano-Se and these metabolite combinations were tested the defense-related compounds to Sitobion avenae in wheat plants. The combination of nano-Se and MT achieved the best controls in reducing the $S$. avenae number. The concentrations of antioxidants, phenolic acids, flavonoids, and plant hormones were found to be markedly enhanced by acting synergistically of nano-Se and MT combination. The VOCs could function as insect attractants and repellents, which included ethanol, 1-popanol, isopentyl alcohol, 2-butanone, 2-heptanone, acetoin, acetone, benzaldehyde, ethyl-2methylpropanoate, and ethyl acetate dimer. Therefore, the application of the optimal ratio of nano-Se and MT could be a very promising strategy to protect wheat seedlings from $S$. avenae infection by collective actions from the secondary metabolic pathways and contributions of the increasing antioxidants and VOCs levels.

\section{Supporting Information}

A: wheat height. B: root length. C: fresh weight of wheat (Figure S1); A: luteolin. B: quercetin (Figure S2); A: caffeic acid. B: ferulic acid. C: chlorogenic acid. D: 4hydroxy-3,5-dimethoxycinnamic acid. E: 4-hydroxycinnamic acid (Figure S3); A: jasmonic acid. B: salicylic acid. C: DIMBOA. D: melatonin (Figure S4); HPLCMS/MS parameters of phytohormones (Table S1); HPLC-MS/MS parameters of flavone compounds (Table S2); HPLC-MS/MS parameters of phenolic acids (Table S3); Analysis conditions of GC-IMS unit (Table S4); Gas chromatographic conditions (Table S5); Primer of RT-qPCR for Genes Involved in DIMBOA and MT Responses (Table S6); Qualitative compounds listed by GC-IMS analysis (Table S7). 


\section{Credit authorship contribution statement}

470 Chunran Zhou: Methodology, Data curation, Investigation, Visualization, Formal analysis, Writing-original draft. Dong Li: Writing-original draft, Investigation, Formal

472 analysis, Data curation. Xinlei Shi: Conceptualization, Methodology. Jingbang

473 Zhang: Validation, Investigation. Quanshun An: Writing-review \& editing. Yangliu

474 Wu: Conceptualization, Methodology. Lu Kang: Methodology, Investigation. Jia-Qi

475 Li: Conceptualization. Canping Pan: Supervision.

\section{Acknowledgments}

Funding: This work was supported by the National Nature Science Foundation of China

479 (3217170041).

\section{Availability of data and materials}

All data generated or analysed during this study are included in this published article within each editable graph

\section{Declarations}

486 Ethics approval and consent to participate

487 Not applicable.

\section{Consent for publication}

489 Not applicable.

\section{Competing interests}

491 The authors declare that they have no known competing financial interests or personal 492 relationships that could have appeared to influence the work reported in this paper.

\section{Reference}

495 [1] T. Zust, A.A. Agrawal, Mechanisms and evolution of plant resistance to aphids, Nat Plants 2 496 (2016) 15206. https://doi.org/10.1038/nplants.2015.206. 
[2] Y. Xu, S.M. Gray, Aphids and their transmitted potato viruses: A continuous challenges in potato crops, Journal of Integrative Agriculture 19(2) (2020) 367-375.

https://doi.org/10.1016/s2095-3119(19)62842-x.

[3] Y.-F. Li, J.-J. An, Z.-H. Dang, W.-L. Pan, Z.-L. Gao, Systemic control efficacy of neonicotinoids seeds dressing on English grain aphid (Hemiptera: Aphididae), Journal of AsiaPacific Entomology 21(1) (2018) 430-435. https://doi.org/10.1016/j.aspen.2018.01.003. [4] A. Mansion-Vaquié, A. Wezel, A. Ferrer, Wheat genotypic diversity and intercropping to control cereal aphids, Agriculture, Ecosystems \& Environment 285 (2019) 106604. https://doi.org/10.1016/j.agee.2019.106604.

[5] A. Ali, N. Desneux, Y. Lu, K. Wu, Key aphid natural enemies showing positive effects on wheat yield through biocontrol services in northern China, Agriculture, Ecosystems \& Environment 266 (2018) 1-9. https://doi.org/10.1016/j.agee.2018.07.012. [6] S. Jacques, J. Reidy-Crofts, J. Sperschneider, L.G. Kamphuis, L.L. Gao, O.R. Edwards, K.B. Singh, An RNAi supplemented diet as a reverse genetics tool to control bluegreen aphid, a major pest of legumes, Sci Rep 10(1) (2020) 1604. https://doi.org/10.1038/s41598-020-58442-4. [7] J. He, Y. Liu, D. Yuan, M. Duan, Y. Liu, Z. Shen, C. Yang, Z. Qiu, D. Liu, P. Wen, J. Huang, D. Fan, S. Xiao, Y. Xin, X. Chen, L. Jiang, H. Wang, L. Yuan, J. Wan, An R2R3 MYB transcription factor confers brown planthopper resistance by regulating the phenylalanine ammonia-lyase pathway in rice, Proc Natl Acad Sci U S A 117(1) (2020) 271-277.

https://doi.org/10.1073/pnas.1902771116.

[8] X. Qi, M. Chen, D. Liang, Q. Xu, F. Zhou, X. Chen, Jasmonic acid, ethylene and ROS are involved in the response of cucumber (Cucumis sativus L.) to aphid infestation, Scientia Horticulturae 269 (2020) 109421. https://doi.org/10.1016/j.scienta.2020.109421.

[9] P. Czerniewicz, H. Sytykiewicz, R. Durak, B. Borowiak-Sobkowiak, G. Chrzanowski, Role of phenolic compounds during antioxidative responses of winter triticale to aphid and beetle attack, Plant Physiol Biochem 118 (2017) 529-540. https://doi.org/10.1016/j.plaphy.2017.07.024. [10] S. Varsani, S. Grover, S. Zhou, K.G. Koch, P.C. Huang, M.V. Kolomiets, W.P. Williams, T. Heng-Moss, G. Sarath, D.S. Luthe, G. Jander, J. Louis, 12-Oxo-Phytodienoic Acid Acts as a Regulator of Maize Defense against Corn Leaf Aphid, Plant Physiol 179(4) (2019) 1402-1415. https://doi.org/10.1104/pp.18.01472.

[11] H.M. NIEMEYER, Hydroxamic Acids Derived from 2-Hydroxy-2H-1,4-Benzoxazin-3(4H)one: Key Defense Chemicals of Cereals, J. Agric. Food Chem. 57 (2009) 1677-1696.

[12] L.M. HANSEN, Effect of 6-Methoxybenzoxazolin-2-one (MBOA) on the Reproduction Rate of the Grain Aphid (Sitobion avenae F.), J. Agric. Food Chem. 54 (2006) 1031-1035.

[13] K.M. Chapman, L. Marchi-Werle, T.E. Hunt, T.M. Heng-Moss, J. Louis, Abscisic and Jasmonic Acids Contribute to Soybean Tolerance to the Soybean Aphid (Aphis glycines Matsumura), Sci Rep 8(1) (2018) 15148. https://doi.org/10.1038/s41598-018-33477-w. [14] G. Bapat, S. Zinjarde, V. Tamhane, Evaluation of silica nanoparticle mediated delivery of protease inhibitor in tomato plants and its effect on insect pest Helicoverpa armigera, Colloids Surf B Biointerfaces 193 (2020) 111079. https://doi.org/10.1016/j.colsurfb.2020.111079. [15] P. Ahmad, E.F. Abd Allah, A. Hashem, M. Sarwat, S. Gucel, Exogenous Application of Selenium Mitigates Cadmium Toxicity in Brassica juncea L. (Czern \& Cross) by Up-Regulating Antioxidative System and Secondary Metabolites, Journal of Plant Growth Regulation 35(4) (2016) 936-950. https://doi.org/10.1007/s00344-016-9592-3. 
[16] X. Zhang, L. Chen, R. Leng, J. Zhang, Y. Zhou, Y. Zhang, S. Yang, K. He, B. Huang, Mechanism study of the beneficial effect of sodium selenite on metabolic disorders in imidacloprid-treated garlic plants, Ecotoxicol Environ Saf 200 (2020) 110736. https://doi.org/10.1016/j.ecoenv.2020.110736.

[17] Y. Li, N. Zhu, X. Liang, L. Zheng, C. Zhang, Y.F. Li, Z. Zhang, Y. Gao, J. Zhao, A comparative study on the accumulation, translocation and transformation of selenite, selenate, and SeNPs in a hydroponic-plant system, Ecotoxicol Environ Saf 189 (2020) 109955.

https://doi.org/10.1016/j.ecoenv.2019.109955.

[18] S.M. Zahedi, M. Abdelrahman, M.S. Hosseini, N.F. Hoveizeh, L.P. Tran, Alleviation of the effect of salinity on growth and yield of strawberry by foliar spray of selenium-nanoparticles, Environ Pollut 253 (2019) 246-258. https://doi.org/10.1016/j.envpol.2019.04.078.

[19] J. Xu, W. Jia, C. Hu, M. Nie, J. Ming, Q. Cheng, M. Cai, X. Sun, X. Li, X. Zheng, J. Wang, $\mathrm{X}$. Zhao, Selenium as a potential fungicide could protect oilseed rape leaves from Sclerotinia sclerotiorum infection, Environ Pollut 257 (2020) 113495.

https://doi.org/10.1016/j.envpol.2019.113495.

[20] J.L.F. Colin F Quinn1†, 2,4†, Ray JB Reynolds1, Jennifer J Cappa1, Sirine C Fakra3, Matthew A Marcus3, Stormy D Lindblom1, Erin K Quinn1, Lindsay E Bennett1, Elizabeth AH Pilon-Smits ${ }^{*}$, Selenium hyperaccumulation offers protection from cell disruptor herbivores, BMC Ecology (2010) 1472-6785.

[21] M.Q. Li, M.K. Hasan, C.X. Li, G.J. Ahammed, X.J. Xia, K. Shi, Y.H. Zhou, R.J. Reiter, J.Q. $\mathrm{Yu}, \mathrm{M} . X . \mathrm{Xu}, \mathrm{J}$. Zhou, Melatonin mediates selenium-induced tolerance to cadmium stress in tomato plants, J Pineal Res 61(3) (2016) 291-302. https://doi.org/10.1111/jpi.12346.

[22] Z. Ulhassan, Q. Huang, R.A. Gill, S. Ali, T.M. Mwamba, B. Ali, F. Hina, W. Zhou, Protective mechanisms of melatonin against selenium toxicity in Brassica napus: insights into physiological traits, thiol biosynthesis and antioxidant machinery, BMC Plant Biol 19(1) (2019) 507. https://doi.org/10.1186/s12870-019-2110-6.

[23] A.R. War, H.C. Sharma, M.G. Paulraj, M.Y. War, S. Ignacimuthu, Herbivore induced plant volatiles: their role in plant defense for pest management, Plant Signal Behav 6(12) (2011) 19731978. https://doi.org/10.4161/psb.6.12.18053.

[24] P. Mitra, S. Das, R. Debnath, S.H. Mobarak, A. Barik, Identification of Lathyrus sativus plant volatiles causing behavioral preference of Aphis craccivora, Pest Manag Sci 77(1) (2021) 285299. https://doi.org/10.1002/ps.6018.

[25] Y. Xiao, Q. Wang, M. Erb, T.C. Turlings, L. Ge, L. Hu, J. Li, X. Han, T. Zhang, J. Lu, G. Zhang, Y. Lou, Specific herbivore-induced volatiles defend plants and determine insect community composition in the field, Ecol Lett 15(10) (2012) 1130-1139. https://doi.org/10.1111/j.1461-0248.2012.01835.x.

[26] K.J. Delaney, M. Wawrzyniak, G. Lemanczyk, D. Wrzesinska, D. Piesik, Synthetic cisjasmone exposure induces wheat and barley volatiles that repel the pest cereal leaf beetle, Oulema melanopus L, J Chem Ecol 39(5) (2013) 620-629. https://doi.org/10.1007/s10886-013-0281-4. [27] R.S. de Oliveira, M. Penaflor, F.G. Goncalves, M.V. Sampaio, A.P. Korndorfer, W.D. Silva, J.M.S. Bento, Silicon-induced changes in plant volatiles reduce attractiveness of wheat to the bird cherry-oat aphid Rhopalosiphum padi and attract the parasitoid Lysiphlebus testaceipes, PLoS One 15(4) (2020) e0231005. https://doi.org/10.1371/journal.pone.0231005.

[28] J. Liu, X. Zhao, Y. Zhan, K. Wang, F. Francis, Y. Liu, New Slow Release Mixture of (E)-beta- 
farnesene with Methyl Salicylate to Enhance Aphid Biocontrol Efficacy in Wheat Ecosystem, Pest Manag Sci (2021). https://doi.org/10.1002/ps.6378.

[29] S. Mechora, Selenium as a Protective Agent Against Pests: A Review, Plants 8(8) (2019) 262. https://doi.org/10.3390/plants8080262.

[30] D. Li, C. Zhou, N. Zou, Y. Wu, J. Zhang, Q. An, J.Q. Li, C. Pan, Nanoselenium foliar application enhances biosynthesis of tea leaves in metabolic cycles and associated responsive pathways, Environ Pollut 273 (2021) 116503. https://doi.org/10.1016/j.envpol.2021.116503. [31] D. Li, Q. An, Y. Wu, J.-Q. Li, C. Pan, Foliar Application of Selenium Nanoparticles on Celery Stimulates Several Nutrient Component Levels by Regulating the $\alpha$-Linolenic Acid Pathway, ACS Sustainable Chemistry \& Engineering 8(28) (2020) 10502-10510.

https://doi.org/10.1021/acssuschemeng.0c02819.

[32] C.H. Kong, S.Z. Zhang, Y.H. Li, Z.C. Xia, X.F. Yang, S.J. Meiners, P. Wang, Plant neighbor detection and allelochemical response are driven by root-secreted signaling chemicals, Nat Commun 9(1) (2018) 3867. https://doi.org/10.1038/s41467-018-06429-1.

[33] M.A. Jamieson, L.A. Burkle, J.S. Manson, J.B. Runyon, A.M. Trowbridge, J. Zientek, Global change effects on plant-insect interactions: the role of phytochemistry, Curr Opin Insect Sci 23 (2017) 70-80. https://doi.org/10.1016/j.cois.2017.07.009.

[34] A.F. El Mehdawi, C.F. Quinn, E.A. Pilon-Smits, Selenium hyperaccumulators facilitate selenium-tolerant neighbors via phytoenrichment and reduced herbivory, Curr Biol 21(17) (2011) 1440-9. https://doi.org/10.1016/j.cub.2011.07.033.

[35] B. Hawrylak-Nowak, M. Hasanuzzaman, R. Matraszek-Gawron, Mechanisms of SeleniumInduced Enhancement of Abiotic Stress Tolerance in Plants, Plant Nutrients and Abiotic Stress Tolerance (2018) 269-295. https://doi.org/10.1007/978-981-10-9044-8 12. [36] D. Li, C. Zhou, J. Zhang, Q. An, Y. Wu, J.Q. Li, C. Pan, Nanoselenium Foliar Applications Enhance the Nutrient Quality of Pepper by Activating the Capsaicinoid Synthetic Pathway, J Agric Food Chem 68(37) (2020) 9888-9895. https://doi.org/10.1021/acs.jafc.0c03044. [37] W. Wang, P. Zhou, X. Mo, L. Hu, N. Jin, X. Chen, Z. Yu, J. Meng, M. Erb, Z. Shang, A.M.R. Gatehouse, J. Wu, Y. Lou, Induction of defense in cereals by 4-fluorophenoxyacetic acid suppresses insect pest populations and increases crop yields in the field, Proc Natl Acad Sci U S A 117(22) (2020) 12017-12028. https://doi.org/10.1073/pnas.2003742117.

[38] A.A. Elkelish, M.H. Soliman, H.A. Alhaithloul, M.A. El-Esawi, Selenium protects wheat seedlings against salt stress-mediated oxidative damage by up-regulating antioxidants and osmolytes metabolism, Plant Physiol Biochem 137 (2019) 144-153.

https://doi.org/10.1016/j.plaphy.2019.02.004.

\section{[39] L. Jiang, L.L. Peng, Y.Y. Cao, K. Thakur, F. Hu, S.M. Tang, Z.J. Wei, Effect of Dietary} Selenium Supplementation on Growth and Reproduction of Silkworm Bombyx mori L, Biol Trace Elem Res 193(1) (2020) 271-281. https://doi.org/10.1007/s12011-019-01690-X.

[40] F. Scheys, K. De Schutter, K. Subramanyam, E.J.M. Van Damme, G. Smagghe, Protection of rice against Nilaparvata lugens by direct toxicity of sodium selenate, Arch Insect Biochem Physiol 103(3) (2020) e21644. https://doi.org/10.1002/arch.21644. [41] Z. Xu, C. Qi, M. Zhang, J. Zhu, J. Hu, K. Feng, J. Sun, P. Wei, G. Shen, P. Zhang, L. He, Selenium mediated host plant-mite conflict: defense and adaptation, Pest Manag Sci (2021). https://doi.org/10.1002/ps.6337.

[42] B. Hanson, S.D. Lindblom, M.L. Loeffler, E.A.H. Pilon-Smits, Selenium protects plants from 
phloem-feeding aphids due to both deterrence and toxicity, New Phytologist 162(3) (2004) 655662. https://doi.org/10.1111/j.1469-8137.2004.01067.x.

[43] M. Erb, S. Meldau, G.A. Howe, Role of phytohormones in insect-specific plant reactions, Trends Plant Sci 17(5) (2012) 250-9. https://doi.org/10.1016/j.tplants.2012.01.003. [44] T. Lortzing, A. Steppuhn, Jasmonate signalling in plants shapes plant-insect interaction ecology, Curr Opin Insect Sci 14 (2016) 32-39. https://doi.org/10.1016/j.cois.2016.01.002. [45] P.I. Kerchev, B. Fenton, C.H. Foyer, R.D. Hancock, Plant responses to insect herbivory: interactions between photosynthesis, reactive oxygen species and hormonal signalling pathways, Plant Cell Environ 35(2) (2012) 441-53. https://doi.org/10.1111/j.1365-3040.2011.02399.x. [46] D.K. Tripathi, K. Vishwakarma, V.P. Singh, V. Prakash, S. Sharma, S. Muneer, M. Nikolic, R. Deshmukh, M. Vaculik, F.J. Corpas, Silicon crosstalk with reactive oxygen species, phytohormones and other signaling molecules, J Hazard Mater 408 (2021) 124820.

https://doi.org/10.1016/j.jhazmat.2020.124820.

[47] M. Barbera, L. Escriva, J.M. Collantes-Alegre, G. Meca, E. Rosato, D. Martinez-Torres, Melatonin in the seasonal response of the aphid Acyrthosiphon pisum, Insect Sci 27(2) (2020) 224-238. https://doi.org/10.1111/1744-7917.12652. [48] C. Liu, L. Chen, R. Zhao, R. Li, S. Zhang, W. Yu, J. Sheng, L. Shen, Melatonin Induces Disease Resistance to Botrytis cinerea in Tomato Fruit by Activating Jasmonic Acid Signaling Pathway, J Agric Food Chem 67(22) (2019) 6116-6124. https://doi.org/10.1021/acs.jafc.9b00058. [49] M.B. Arnao, J. Hernandez-Ruiz, Melatonin: A New Plant Hormone and/or a Plant Master Regulator?, Trends Plant Sci 24(1) (2019) 38-48. https://doi.org/10.1016/j.tplants.2018.10.010. [50] R.K. Tiwari, M.K. Lal, K.C. Naga, R. Kumar, K.N. Chourasia, S. S, D. Kumar, S. Sharma, Emerging roles of melatonin in mitigating abiotic and biotic stresses of horticultural crops, Scientia Horticulturae 272 (2020) 109592. https://doi.org/10.1016/j.scienta.2020.109592. [51] L.N. Meihls, V. Handrick, G. Glauser, H. Barbier, H. Kaur, M.M. Haribal, A.E. Lipka, J. Gershenzon, E.S. Buckler, M. Erb, T.G. Kollner, G. Jander, Natural variation in maize aphid resistance is associated with 2,4-dihydroxy-7-methoxy-1,4-benzoxazin-3-one glucoside methyltransferase activity, Plant Cell 25(6) (2013) 2341-55.

https://doi.org/10.1105/tpc.113.112409.

[52] A. Clavijo McCormick, S.B. Unsicker, J. Gershenzon, The specificity of herbivore-induced plant volatiles in attracting herbivore enemies, Trends Plant Sci 17(5) (2012) 303-10.

https://doi.org/10.1016/j.tplants.2012.03.012.

[53] J. Park, J.A. Thomasson, Z. Gorman, M.J. Brewer, W.L. Rooney, M.V. Kolomiets, Multivariate analysis of sorghum volatiles for the fast screening of sugarcane aphid infestation, Journal of Asia-Pacific Entomology 23(4) (2020) 901-908. https://doi.org/10.1016/j.aspen.2020.07.014. [54] X. Zhuang, A. Fiesselmann, N. Zhao, H. Chen, M. Frey, F. Chen, Biosynthesis and emission of insect herbivory-induced volatile indole in rice, Phytochemistry 73(1) (2012) 15-22. https://doi.org/10.1016/j.phytochem.2011.08.029. [55] Y. Zhang, B. Teng, D. Wang, J. Jiang, Discovery of a specific volatile substance from rice grain and its application in controlling stored-grain pests, Food Chem 339 (2021) 128014. https://doi.org/10.1016/j.foodchem.2020.128014. 
672 Table 1. Numbers of $S$. avenae in the control and the treatments at different

673 concentrations of Nano-Se.

674

\begin{tabular}{ccccc}
\hline $\begin{array}{c}\text { Days after aphid } \\
\text { infection }\end{array}$ & CK & Nano-Se-5mg/L & Nano-Se-10mg/L & Nano-Se-20mg/L \\
\hline 5 & $48.3 \pm 3.1 \mathrm{a}$ & $26.7 \pm 2.5 \mathrm{~d}$ & $33.7 \pm 3.8 \mathrm{c}$ & $41.0 \pm 3.6 \mathrm{~b}$ \\
10 & $126.3 \pm 8.0 \mathrm{a}$ & $87.7 \pm 3.1 \mathrm{c}$ & $97.3 \pm 4.0 \mathrm{c}$ & $112.7 \pm 7.6 \mathrm{~b}$ \\
15 & $463.0 \pm 12.3 \mathrm{a}$ & $307.0 \pm 8.5 \mathrm{~d}$ & $340.7 \pm 12.1 \mathrm{c}$ & $418 \pm 17.0 \mathrm{~b}$ \\
\hline
\end{tabular}

675

676 Data were compared statistically by one-way analysis of variance (ANOVA) and least

677 significant difference (LSD) and Levene test in SPSS 26.0. Different letters in each

678 row indicate the significant difference at $p<0.05$.

679

680 


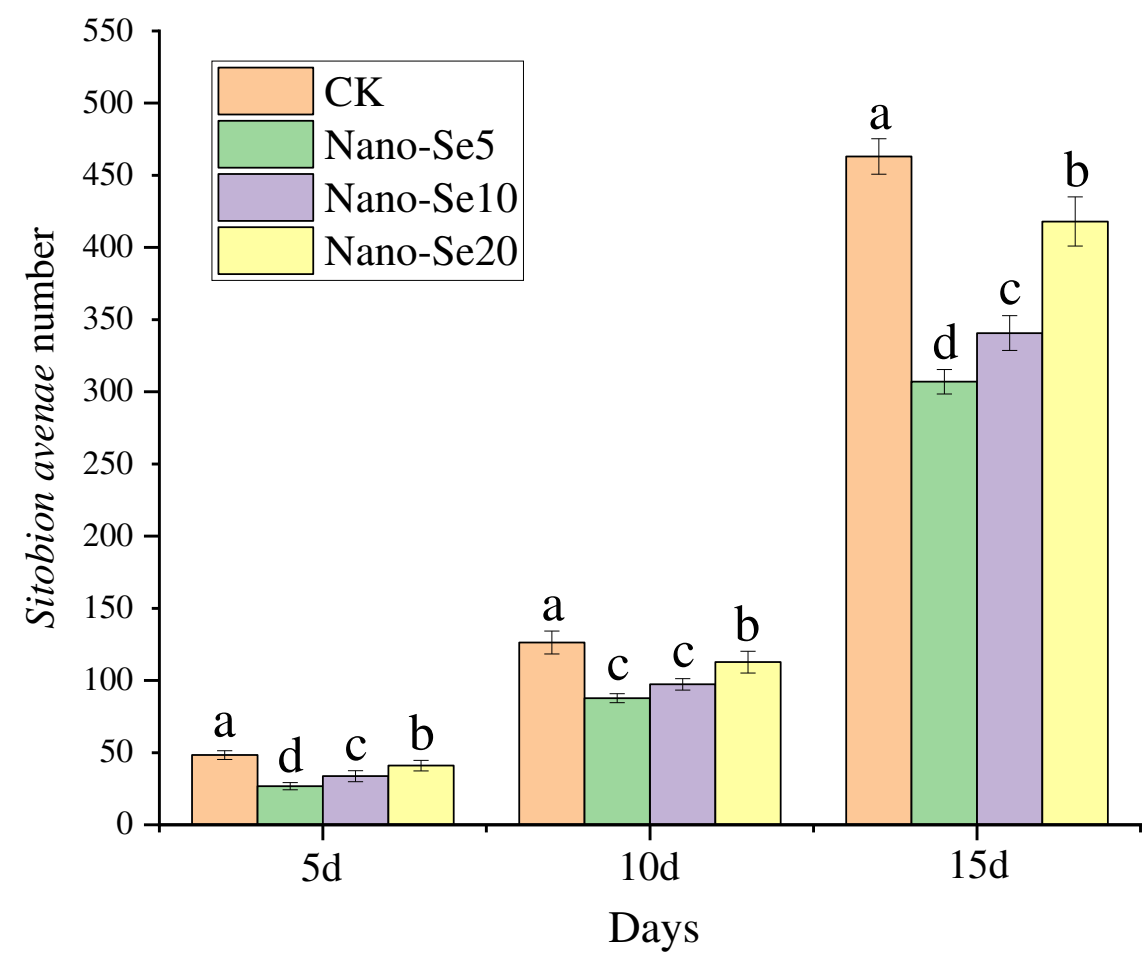

681

682 Figure 1. S. avenae number in control and different concentrations of Nano-Se.

683 Different letters indicate a significant difference at $p<0.05$.

684

685

686 

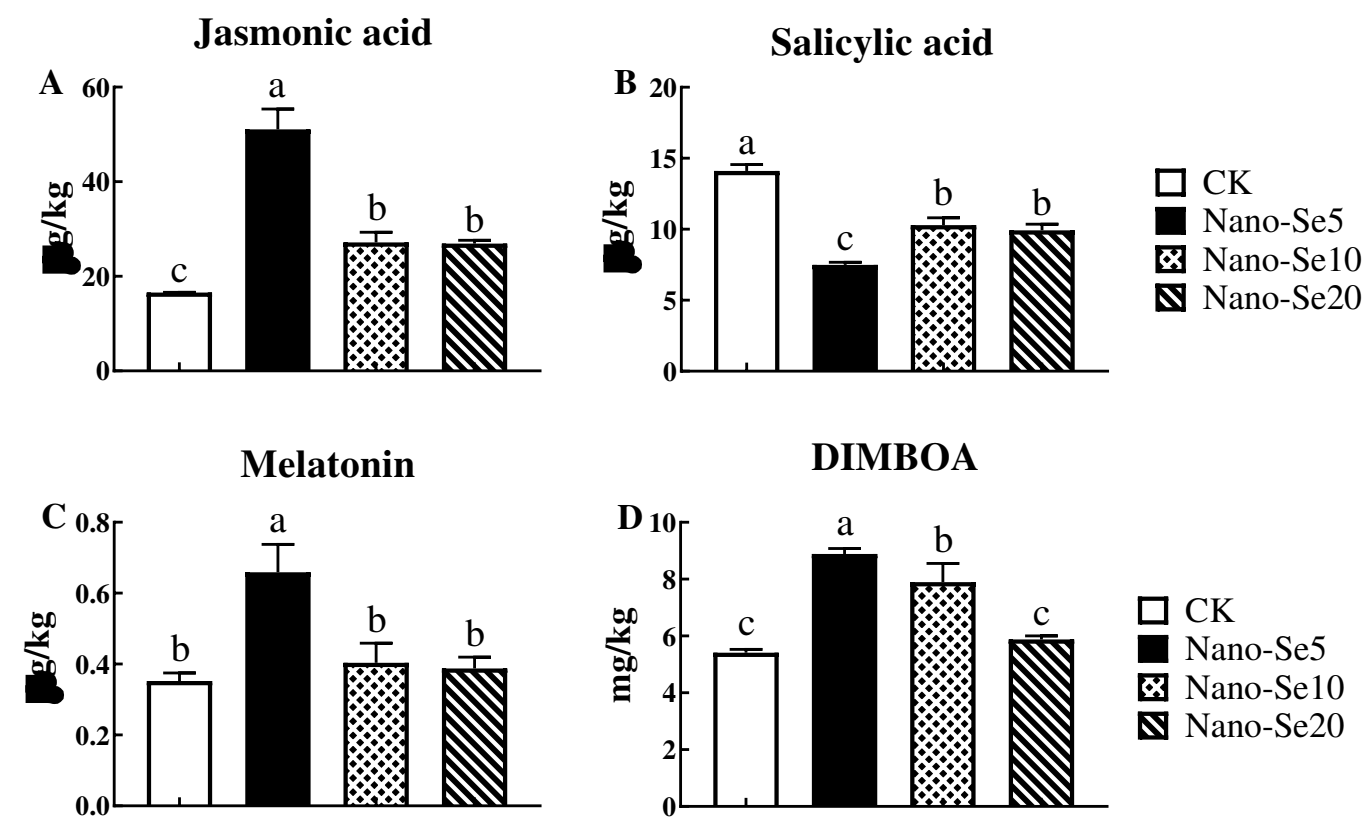

DIMBOA

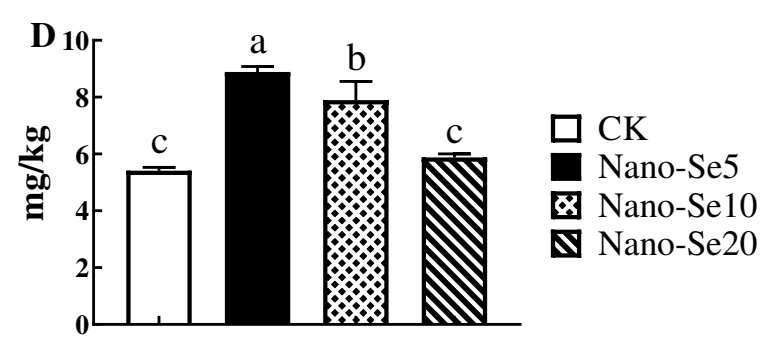

688

689

690 Figure 2. Effects of Nano-Se application on JA(A), SA(B), MT(C), and DIMBOA(D)

691 contents in wheat seedlings. Different letters indicate a significant difference at $692 p<0.05$.

693 

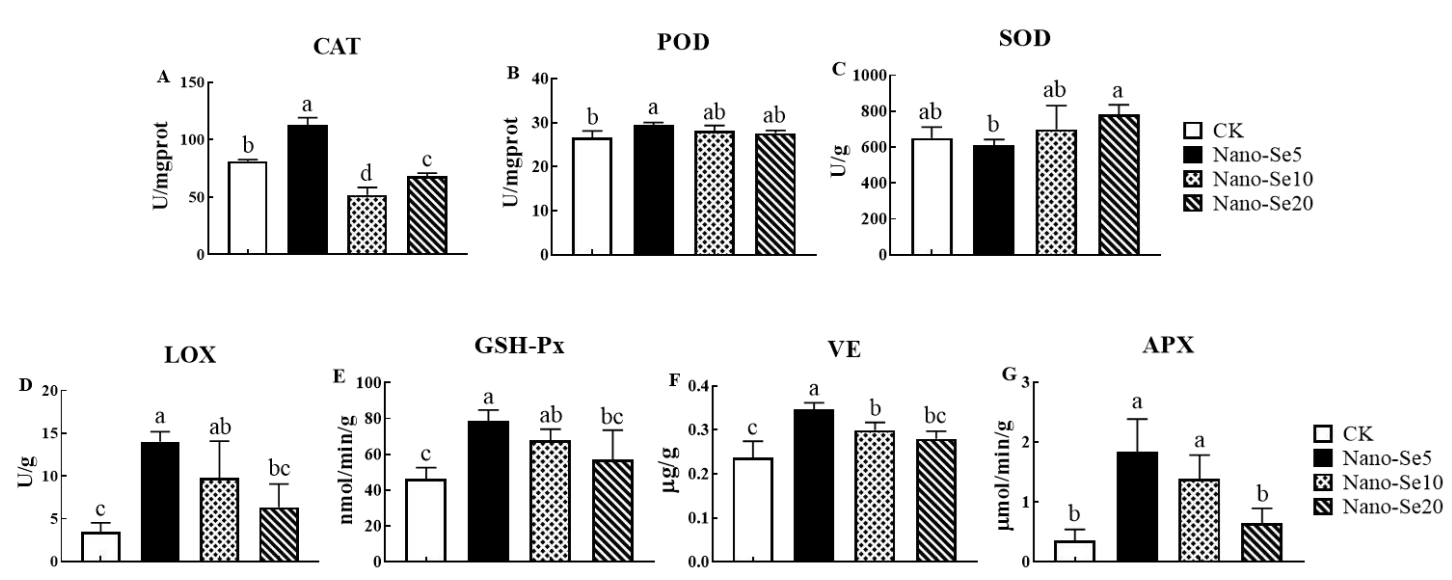

695

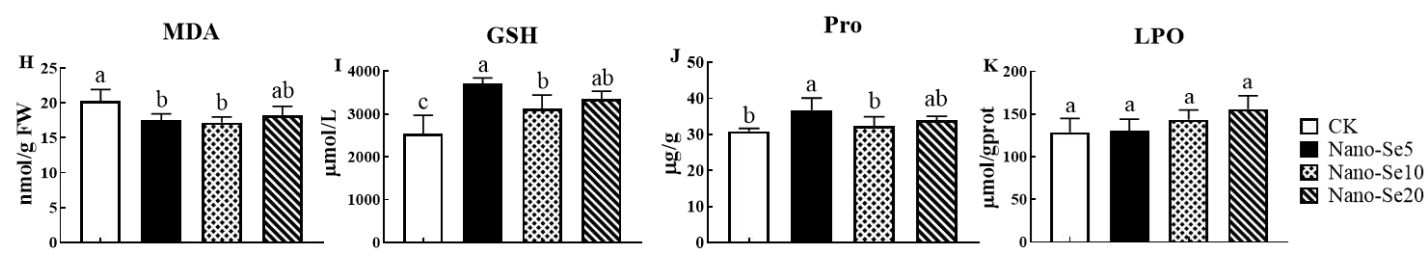

696 Figure 3. Effects of nano-Se application on CAT(A), POD(B), SOD(C), LOX(D),

697 GSH-Px(E), VE(F), APX(G), MDA(H), GSH(I), Pro(J), and LPO(K) contents in

698 wheat seedlings. Different letters in one figure indicate a significant difference at

$699 \quad p<0.05$. 
A
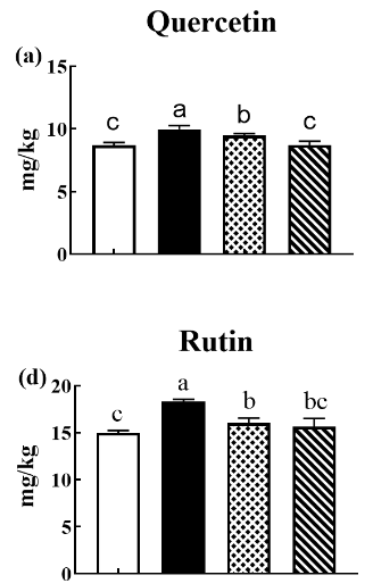
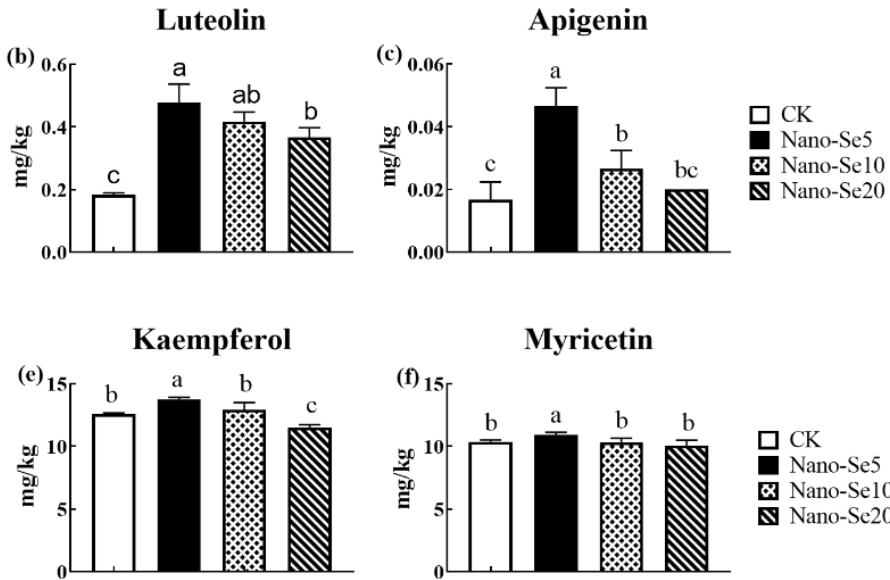

$\mathrm{CK}$

Nano-Se5

Q Nano-Se10
B

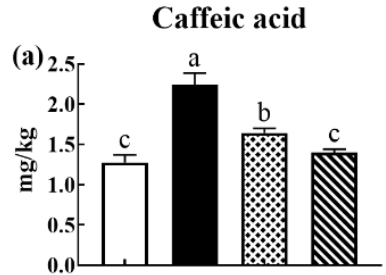

4-Hydroxy-3,5-dimethoxycinnamic acid

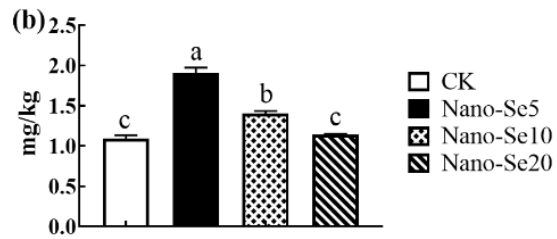

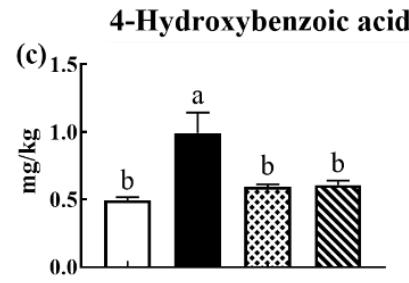
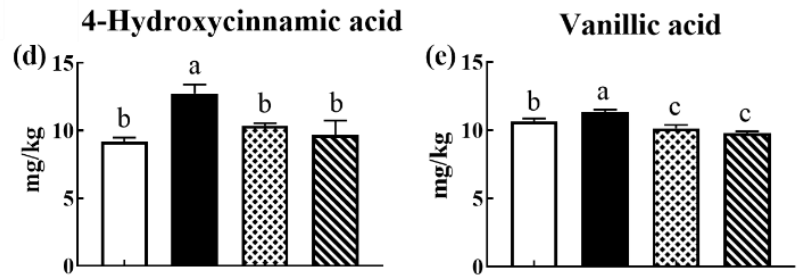

$\square \mathrm{CK}$

Q Nano-Sel0

\$ Nano-Se20
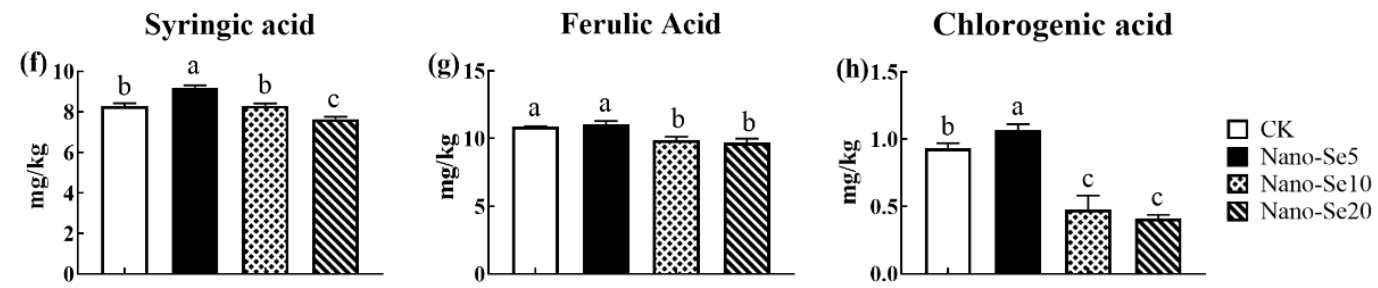

Figure 4. Effects of nano-Se application on the concentration of flavone compounds (A) and phenolic acids (B) in wheat seedlings. Different letters in a figure indicate a significant difference at $p<0.05$. 
A

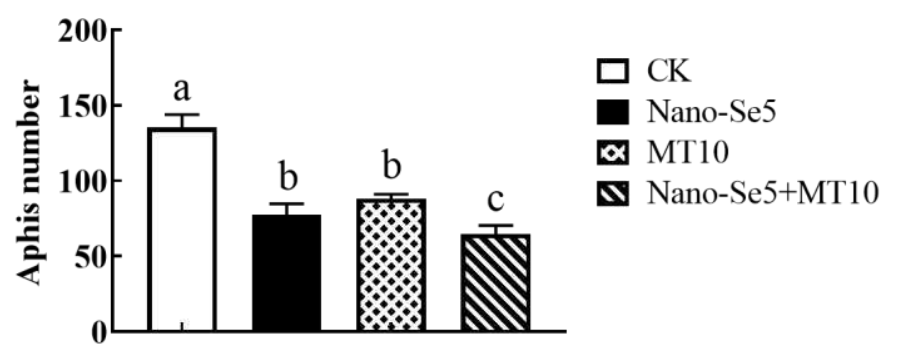

B
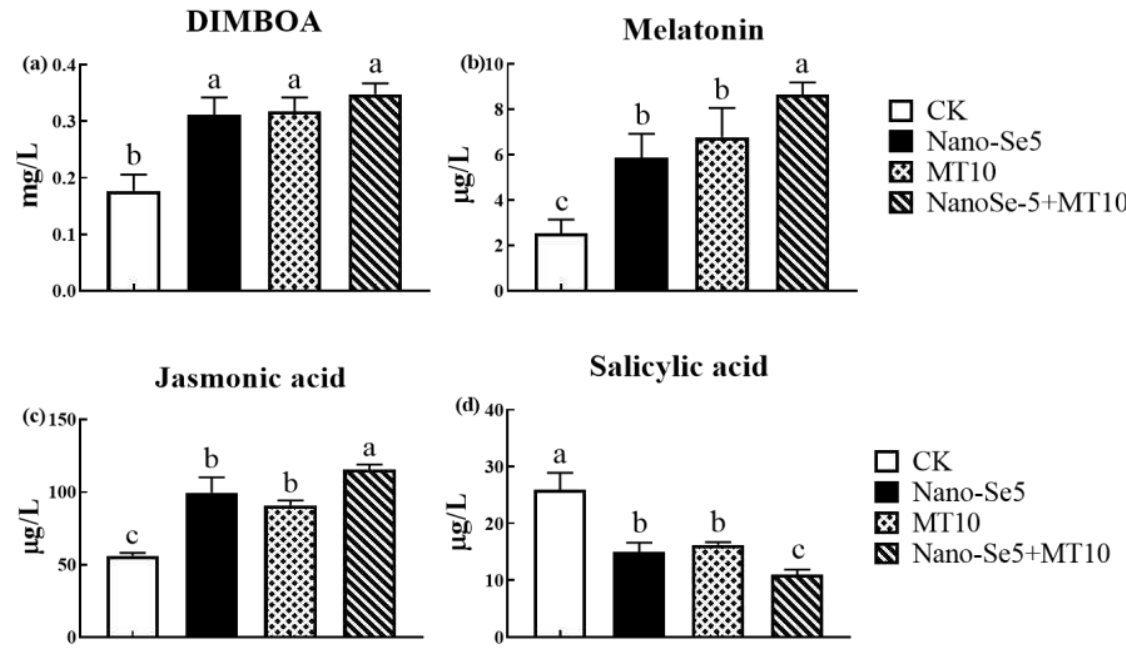

C
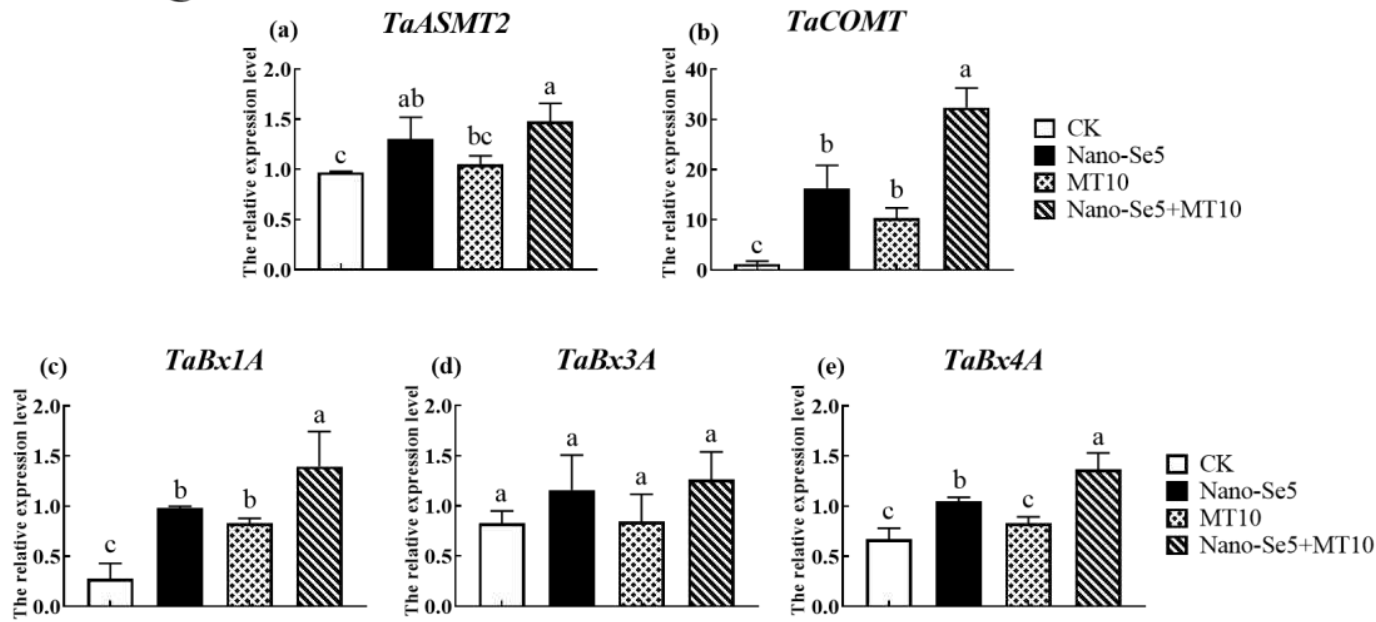

707 Figure 5. Effects of applied nano-Se $(5 \mathrm{mg} / \mathrm{L})$, MT $(10 \mathrm{mg} / \mathrm{L})$ and their combination

708 on S. avenae number (A), DIMBOA, MT, JA, and SA contents (B), and related

709 synthesis genes (C) in wheat seedlings. Different letters among treatments indicate a

710 significant difference at $p<0.05$. 


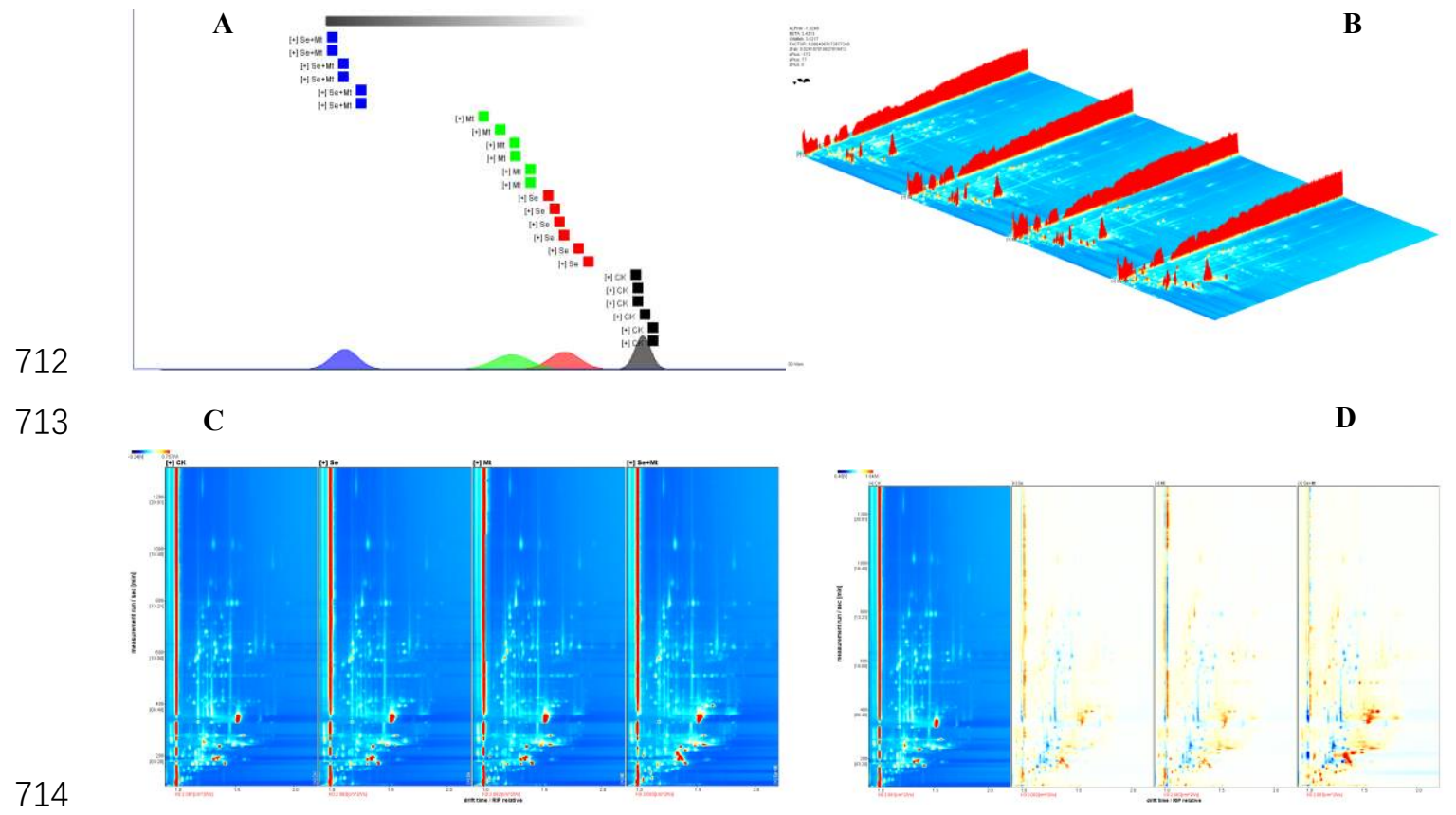

715 Figure 6. Fingerprint similarity analysis (A), GC-IMS three-dimensional spectrum

716 (B), top view (C), and gas phase ion migration spectroscopy(D) of metabolites for all 717 treatment in wheat seedlings with aphid infection. 


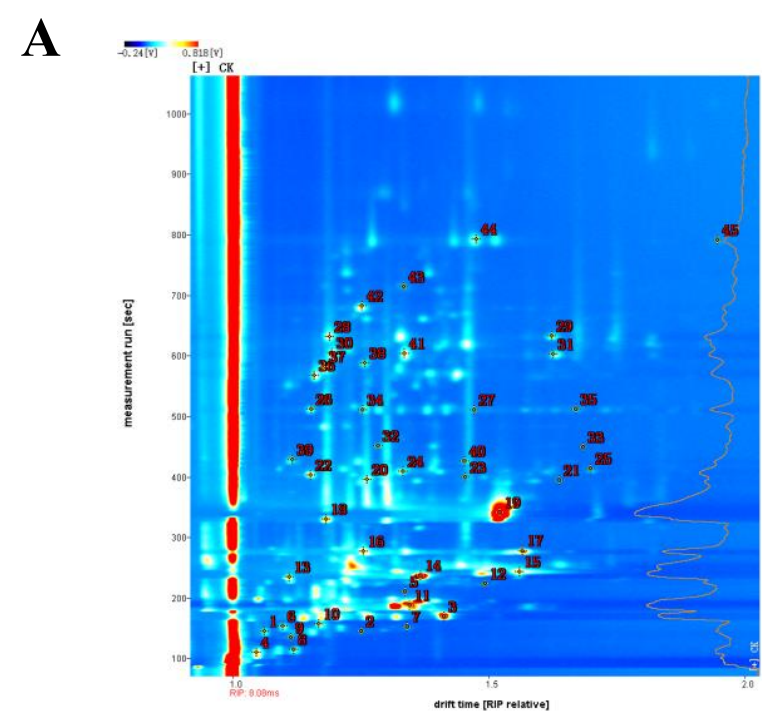

721
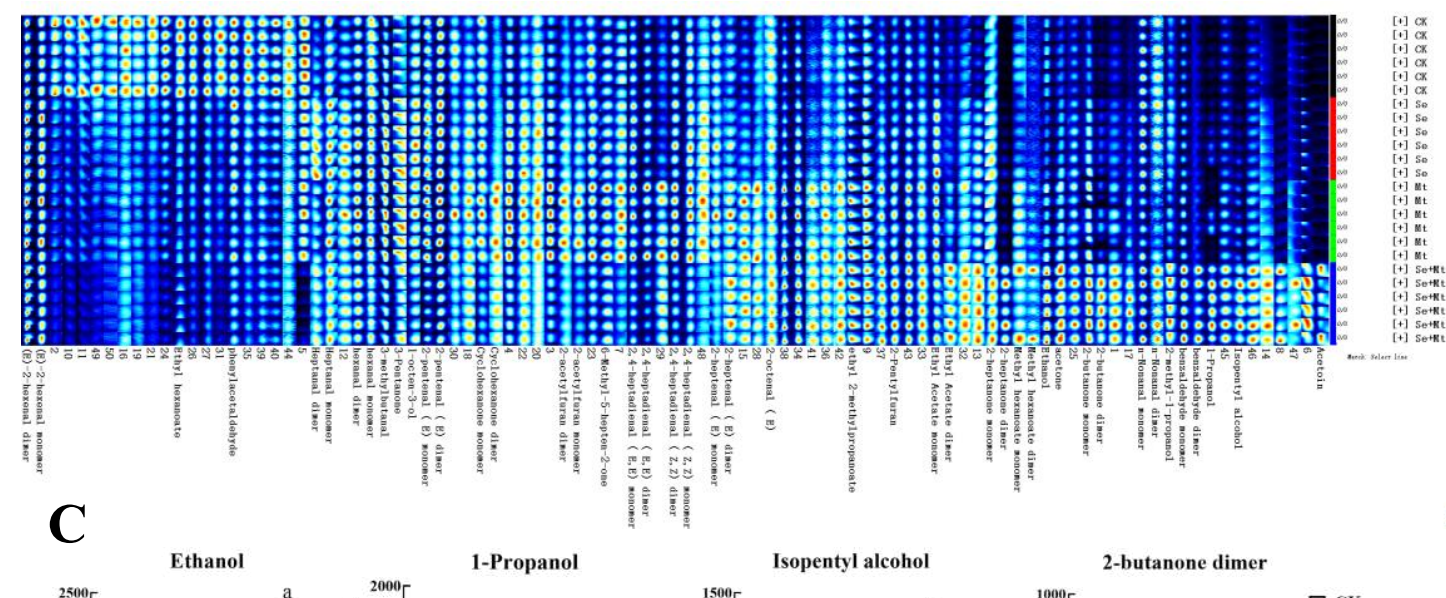

tin
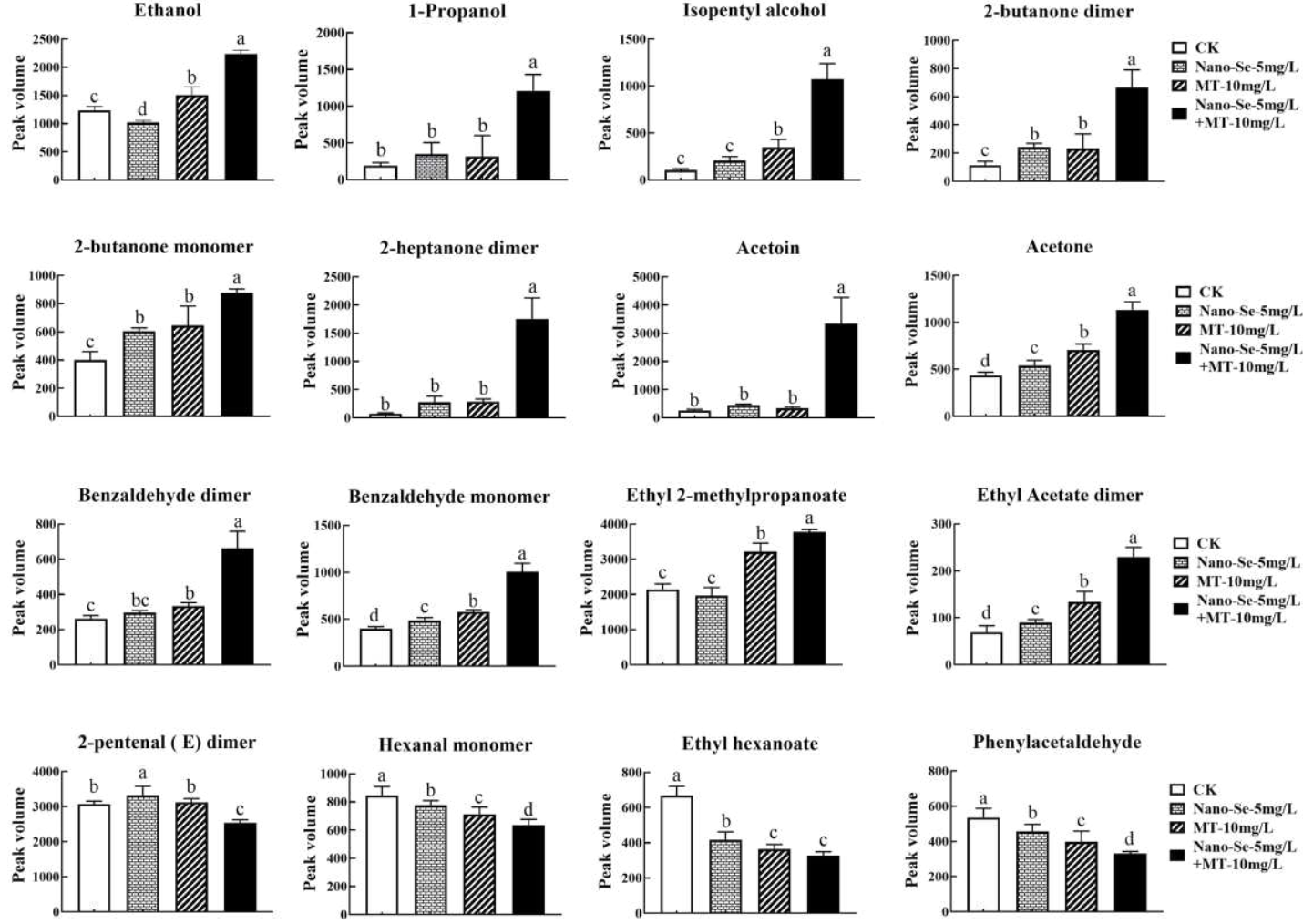
723 Figure 7. Qualitative analysis (A) and gallery plot (B) of volatile organic compounds

724 measured in wheat seedlings. The changes in the concentration of differential

725 metabolites in wheat seedlings exposed to nano-Se $(5 \mathrm{mg} / \mathrm{L})$, melatonin $(10 \mathrm{mg} / \mathrm{L})$,

726 and their combination (C). Different letters among treatments indicate a significant

727 difference at $p<0.05$.

728 


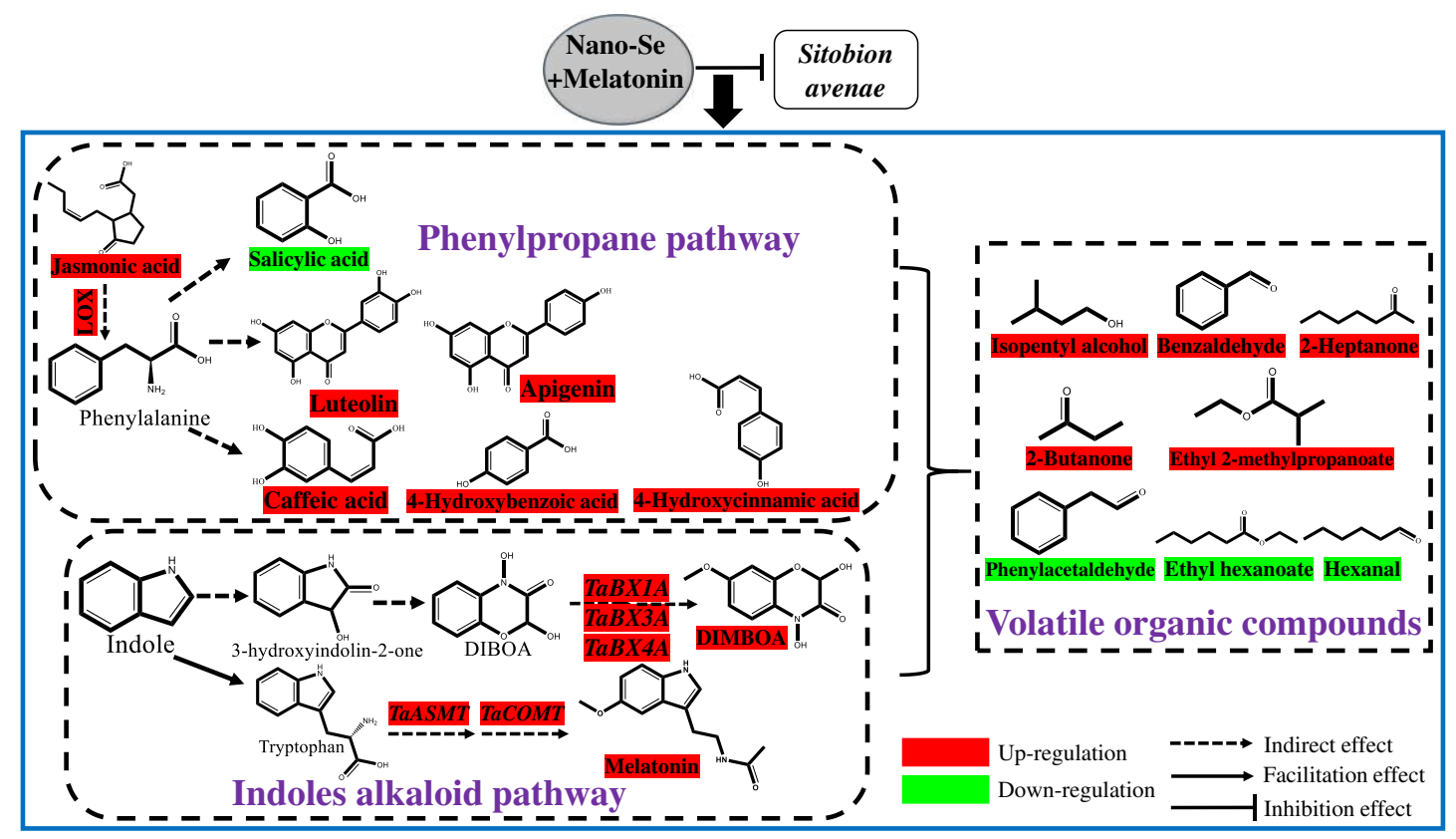

731 Figure 8. Proposed mechanism of nano-Se improving the secondary metabolism in wheat seedlings and against Sitobion avenae infection. 


\section{Supplementary Files}

This is a list of supplementary files associated with this preprint. Click to download.

- Graphicalabstract.docx

- supplementaryinformation.docx 